\title{
Interactive comment on "Radiative transfer acceleration based on the Principal Component Analysis and Look-Up Table of corrections: Optimization and application to UV ozone profile retrievals" by Juseon Bak et al.
}

Juseon Bak et al.

juseonbak@pusan.ac.kr

Received and published: 23 November 2020

The comment was uploaded in the form of a supplement:

https://amt.copernicus.org/preprints/amt-2020-349/amt-2020-349-AC1-

supplement.pdf 